\title{
HUMAN FACTORS FOR STEREOSCOPIC IMAGES
}

\author{
Kazuhiko Ukai
}

\author{
School of Science and Engineering, Waseda University
}

\begin{abstract}
Human factors related to stereoscopic motion images, such as visual stress, are introduced. Following a survey of studies on accommodation and convergence, difficulties in fusing two images, fatigue possibly due to a discrepancy between accommodative and convergence stimuli are introduced. Changes in oculomotor function after viewing stereoscopic images, and irreversible changes in oculomotor functions are also discussed.
\end{abstract}

\section{INTRODUCTION}

Depth perception in human vision relies on a number of cues that are either psychological or physiological in origin. Psychological cues include: perspective, overlap, air perspective, shadow, apparent size, texture, etc. Physiological cues include: binocular parallax and motion parallax. However, depth perception can still be experienced when all physiological cues are missing, otherwise depth would never be perceived while viewing pictures and paintings. In motion images depth is perceived, even when the viewer is not moving, due to a type of motion parallax that arises from camera movement. Three-dimensional computer graphics (3D-CGs) rely mainly on this physiological cue in addition to psychological ones.

Binocular parallax refers in general to image difference, while binocular disparity refers to retinal image differences, due to the small spatial displacement between the left and right eyes, which requires convergence to fuse the image at

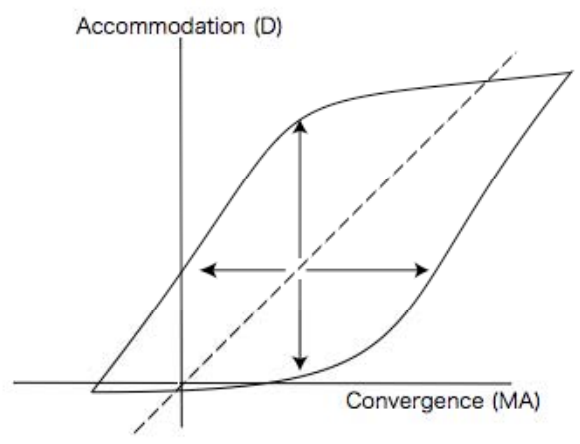

Figure 1. Area where the combination of accommodation and convergence stimuli causes neither blurred nor double vision. The broken line shows the 'Donders line.' a particular distance. Physiologically, binocular disparity evokes a sense of depth. If all cues mentioned above are included in an image, it is a fully 3-D image. Though various projects are underway to create true 3 -D images, an inexpensive system capable of executing this task remains to be developed. To date, stereoscopic images have only been possible to realize easily. One problem is that discrepancy between accommodative and vergence stimuli is common in such images because accommodation should respond only to the screen/image position, but disparity of the two images for both eyes, vergence stimulus, varies with time. This conflict can be highly stressful for the visual system.

\section{ACCOMMODATION AND CONVERGENCE}

Usually, accommodation and convergence responses are closely related. However, they may also differ depending on the stimuli. If the discrepancy is small, the two functions respond correctly. The stimuli and responses are unnatural, and result in visual stress according to the accepted theory. A brief review of the characteristics of accommodation and vergence is presented in this section.

Accommodation has limits for both near and distant viewing. Displacement of the far point of accommodation from infinity is known as ametropia. The near point of accommodation increases due to aging. The resulting subjective feeling of focal inability experienced in daily life is known as presbyopia. When accommodation is completely relaxed, it adapts to the far point. However, when no accommodative stimulus is available, as in darkness or an empty field, accommodation assumes an intermediate position between the far and near points. This is known as tonic accommodation. Tonic accommodation is affected by visual fatigue [1]. With fatigue, visual targets further or closer than the tonic accommodative point do not induce precise accommodation and errors are biased toward the tonic position. Such errors are known as accommodative lead or lag. After accommodating to a different position from the tonic accommodative point, tonic position shifts from the initial position. This is known as accommodative adaptation.

Similarly, the convergence process is bounded by the fusion limit, which usually displays a wider range than the accommodation range, particularly in the aged. This is because the convergence function does not diminish with age, as the accommodative function does. The error in 
convergence, known as fixation disparity, is similar to the accommodative lag or lead. It is small and changes with distance in a complex manner with large inter-individual variations. Vergence state under the no-stimulus condition is known as phoria. The change in phoria, after fixation at a certain distance, is known as phoria adaptation, vergence adaptation, or prism adaptation.

Comparison between accommodation and convergence is based on a scale given in units of reciprocal meters $(1 / \mathrm{m})$. Diopters (D) for accommodation and meter angle (MA) for convergence are units based on this principle. Convergence and accommodation cannot be fully independent of each other. The limitations of both functions are shown in Figure 1. This graph shows a combination of accommodative and convergence stimuli. When viewing this graph horizontally, the convergence limit is calculated, while assuming that the accommodative stimulus remains fixed. The resulting area, where the combining of accommodation and convergence stimuli causes neither blurred nor double vision, shows very large individual differences, which depend on phoria, ametropia and its correction, and the degree of presbyopia.

An accommodative response to a stimulus, in the absence of a stimulus for convergence, such as when one eye is occluded, can elicit convergence responses known as accommodative convergence. The ratio of accommodative convergence to the unit accommodation (AC/A ratio) shows the intensity of accommodative convergence. Convergence accommodation, and the ratio of that to the unit convergence (CA/C ratio), can also be defined similarly. AC/A and $\mathrm{CA} / \mathrm{C}$ ratios are changed by the adaptation of accommodation and vergence. However, it is not easy to present a stimulus to convergence without eliciting accommodation.

Accommodative responses have been measured during the viewing of stereoscopic displays [2]. Accommodative responses mark the equilibrium point between convergencedriven accommodation that pulls toward a position closer to the viewer, and defocus-driven accommodation that pushes it to stay at the screen position. In order to support their hypothesis, the demand of defocus-driven accommodation was reduced by blurring, i.e., by reducing the higher spatial
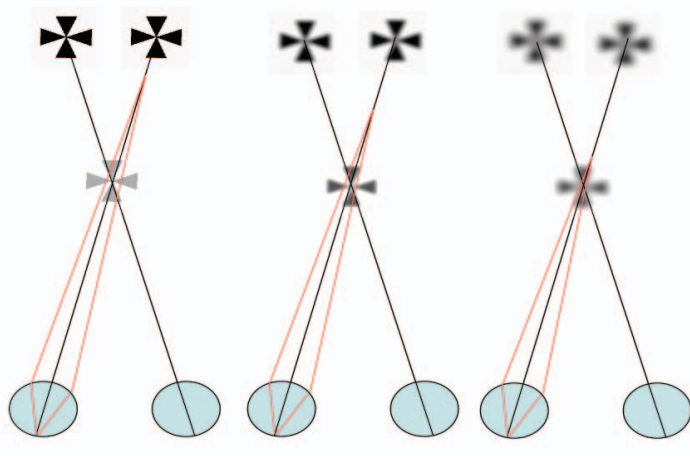

Figure 2. Accommodative responses when viewing stereoscopic images depend on the target blur. Accommodative responses to the stereoscopic images varied by the target blur. frequency components of the visual target. The remaining low spatial frequency components were less affected by defocus, and inaccurate accommodation was permitted. Figure 2 shows an accommodative response that varied with target blurring while viewing stereoscopic images.

The quality of stereoscopic display images is improving continuously, a desirable development. However, when stereoscopic systems were derived, their use increased the occurence of discrepancy between accommodation and convergence, which has lead to possibly increased visual fatigue.

\section{DIFFICULTY TO VIEW STEREOSCOPIC IMAGE}

Sometimes we experience difficulties to fuse binocular images. This phenomenon can be understood from Figure 1. However, the figure changes its form depending on the contents of the image. For example, we cannot fuse the disparities near the fixating position but we can easily fuse the disparities presented at peripheral visual fieald.

Simultaneous measurements of accommodation and convergence when viewing stereoscopic images support to the theory that the cause of visual fatigue is the discrepancy in accommodation and vergence. Recordings revealed unstable accommodative responses as shown in Figure 3 and suggest difficulty in controlling accommodation [3].

\section{FATIGUE/EYE STRAIN}

Visual fatigue can be caused by demands on basic visual functions, such as focusing and converging the eyes on a near object, and may also involve central cortical structures, for example, those involved in processing a wide-field, high-contrast, geometric pattern [4]. Most visual tasks in everyday life can contribute in some way to visual fatigue, especially when the eyes are used for extended periods. Fatigue is increased when doing fine work, reading poorly printed text and low-quality computer images, reading in inadequate or intense lighting, exposure to flickering lights

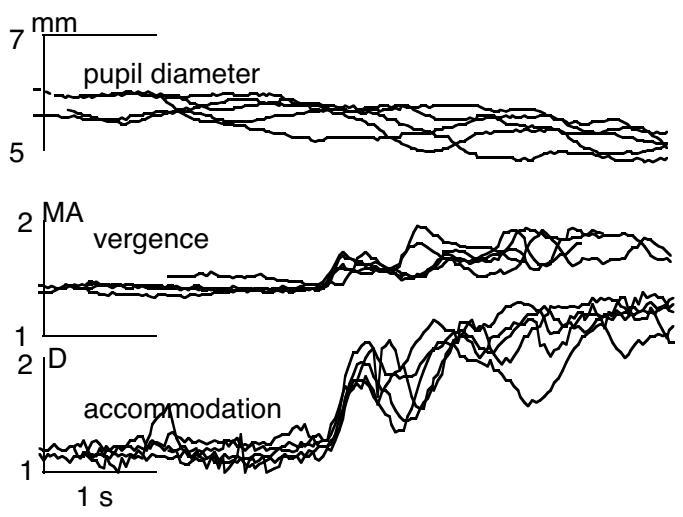

Figure 3. An example of actual recording of near triad (pupil, convergence and accommodation) from a viewer of stereoscopic image after Ukai and Kato [3] with permission by the publisher. 
or geometric patterns, and when a person has uncorrected ametropia. Visual fatigue can also arise when people are viewing stereoscopic motion images. In particular, stereoscopic images used for entertainment should be free of visual fatigue because they are not essential for our lives.

Visual fatigue first became a topic of interest after the 1970's with the introduction of visual display units (VDUs) in the office environment. As a result, international and domestic organizations have published recommendations for working environments using VDUs, in order to reduce fatigue symptoms. In spite of these measures, many people suffer from visual fatigue even today, although it has been reported that the number of people suffering from visual fatigue in the office environment has decreased since the recommendations were implemented.

Symptoms of visual fatigue generally include eyestrain, dried mucus or tears around the eyelids, feelings of pressure in the eyes, aching around the eyes, discomfort when the eyes are open, hot eyes, difficulty in focusing or blurred vision, stiff shoulders, and headaches. Very little is known about the mechanisms of fatigue. This is attributed in part to the fact that fatigue symptoms are largely subjective phenomena. A number of studies have attempted to measure human stress/strain objectively. Among the known studies on visual fatigue, Wilkins has published a book on visual stress [4]. He has proposed that when a person looks at a specific pattern (such as fine stripes), parts of the brain may be stimulated excessively, resulting in migraine and fatigue. Wilkins has suggested that people who develop migraines easily also tend to be affected by eyestrain.

A stereoscopic image is composed of two different images directed to the left and right eyes. Separation of these two images is achieved by several methods [5]. In addition to the fatigue caused by viewing stereoscopic images, features inherent in different viewing methods also cause visual stress among viewers.

Separation by color filters, such as a pair of red and blue glasses is known as an anaglyph. When using this system, color differences in the retinal images of the two eyes cause binocular rivalry. Even though binocular rivalry has been investigated extensively from the perspective of visual system mechanisms, very little is known about its long-term health effects. Polarized filters are widely used in movie theaters and theme park attractions. The effect of cross-talk, when the polarization axes are changed by the tilt of the viewers head, has been pointed out. By a method of time-sharing, using synchronized liquid crystal (LC) shutters, viewers see a flickering image. Flickering is one of the most significant causes of visual fatigue [4]. The parallax barrier system limits freedom of the position from which images can be viewed. The actual arrangement of stereoscopic imaging systems, such as the appropriate shift of the binocular images on the screen, is also a complex issue, especially in a system using two projectors.

Binocular head-mounted displays (HMDs) give rise to certain health problems, even when they are used in a non- stereoscopic (bi-ocular) mode. The axes of the binocular optical system must be controlled precisely in the manufacturing process; otherwise, viewers will experience severe fatigue, especially when both axes are vertically misaligned. When HMDs had axes alignment problems, user fatigue was common. Recently, this has been improved but still causes problems when used for long durations. Stereoscopic HMDs also require strict alignment of axes. Viewers often experience fatigue caused by misalignment in addition to binocular disparity. HMDs have another characteristic. When the head moves, reflex eye movements are provoked. If the visual input is fixed to the head, this eye movement is suppressed [6]. The long-term effects of such suppression on health are as yet unknown. However, generally speaking, imaging with HMDs is still unnatural.

Visual fatigue can be measured by subjective methods. The Simulator Sickness Questionnaire (SSQ) by Kennedy is well established for evaluating motion sickness. We have been using the newly developed questionnaire subjectively to assess symptoms caused by viewing various types of moving images. The questionnaire includes factors on visual fatigue and sickness, and the two factors are clearly distinguished. This scale was used to evaluate four types of moving images: a stereoscopic (anaglyph method)/2-D movie, a TV game using an HMD/TV, images with/without stabilization of camera shake, and a movie with/without color-break-up. Results indicated that stereoscopic movies caused severe visual fatigue compared to other media.

Inter-individual differences in susceptibility to visual fatigue remain unclear. Fatigue symptoms may be a warning of and precursor to serious damage to the human body, resulting from continuous exposure. Thus, severe damage may occur suddenly in those with higher susceptibility. Migraine may be one of the many symptoms of differences in susceptibility to visual fatigue. It is known that repetitive periodic spatial patterns are a cause of visual fatigue and migraine [4] that may cause epileptic seizures in viewers with a diathesis. It is possible that people, who are highly susceptible to migraines, are also easily fatigued by stereoscopic images.

\section{CHANGES IN OCULOMOTOR BALANCE}

A number of studies have investigated the effect of stereoscopic images on various visual functions. In the case of conventional images, a change in binocular eye position with monocular fixation (phoria) may occur due to prism adaptation, convergence adaptation, or phoria adaptation, resulting from continuously viewing an object from a fixed visual distance. With stereoscopic images, an unnatural convergence load may cause these changes. Furthermore, there is also a phenomenon known as accommodative adaptation that is of interest in relation to the progression of myopia. Interactions between the accommodation and the vergence are indicated by accommodative convergence and convergence accommodation. Quantitatively, as discussed 
in the previous section, these functions are referred to as $\mathrm{AC} / \mathrm{A}$ and $\mathrm{CA} / \mathrm{C}$ ratios. They also change following accommodation and vergence adaptations. Thus, viewing stereoscopic images may change the oculomotor balance.

Accommodation is sometimes used as one of the few physiological indicators of fatigue, and thus has been subjected to many types of measurements. Reaction velocity of step responses, especially velocity of disaccommodation and response amplitude, have been used as the main indicators of fatigue. Convergence limit and binocular vision are used when watching stereoscopic images and it is interesting to see how they change as a result of forced convergence caused by fatigue.

Many studies, e.g., [7], have measured changes in these oculomotor and other functions, before and after exposure to image loads. Functions measured were: binocular vision, subjective evaluation of fatigue, far point of accommodation (ametropia), fusional limit, tonic accommodation, velocity of accommodation, phoria, AC/A and $\mathrm{CA} / \mathrm{C}$ ratio, and pupil activities. The types of loads were varied widely: stereoscopic TV images, using the LC shutter system or the parallax barrier system, and stereoscopic HMD/HDTV, for 10,25 , or 30 minutes duration.

Research findings indicated some changes in oculomotor functions. However, results have been inconsistent, probably due to differences in distance between screen and viewer, pop up depths, screen sizes, load durations, presentation systems, and image contents. As a result, the differences in adaptation reported by different studies may be due to differences in experimental protocols. The most significant finding of the studies reviewed above has been that there may be a change in oculomotor balance caused by adaptation of the ocular control systems.

\section{IRREVERSIBLE AFFECTS}

Some ophthalmologists remain concerned that viewing stereoscopic images may cause strabismus in young children. Strabismus is an abnormality in binocular alignment that is usually congenital. It is influenced by accommodation, vergence and binocular vision. There is no evidence for or against the hypothesis that viewing stereoscopic images causes strabismus, except for a report by Tsukuda and Murai [8]. They reported one case of a four years and 11 month old child who manifested esotropia, after viewing stereoscopic animation at a cinema using an anaglyph. Von Noorden [9] described one cause of acute strabismus as a disturbance of binocular vision.

If the visual stress level is too high, or if it continues for a long time, irreversible changes may result. Many studies on myopia have suggested that eyestrain is also a warning of possibly irreversible health damage.

\section{CONCLUSION}

Viewers should be careful to avoid viewing stereoscopic images frequently and for extended durations. Using stereoscopic images with neither a large nor diverging parallax may minimize health problems. Viewers should be prepared to stop immediately, if fusion difficulties are experienced. Hardware/software manufacturers should avoid unnatural image presentations, such as vertical parallax, images that diverge further than infinity, large binocular disparity in the central visual field or around objects that are at the center of the viewer's attention, binocular difference of size or color, as well as distortion between binocular images. Most of these recommendations have also been noted in the ISO document (IWA3) [10]. Further details have been discussed elsewhere [11]. However, a number of issues remain to be addressed in the future.

\section{References}

[1] S. Hasebe, E.W. Graf, and C.M. Schor, "Fatigue Reduces Tonic Accommodation," Ophthalmic and Physiological Optics, vol. 21, pp. 151-60, 2001.

[2] Y. Okada, K. Ukai, J.S. Wolffsohn, B. Gilmartin, A. Iijima, and T. Bando, "Target Spatial Frequency Determines the Response to Conflicting Defocus- and Convergence-Driven Accommodative Stimuli," Vision Research, vol. 46, pp. 475-484, 2006.

[3] K. Ukai and Y. Kato, "The Use of Video Refraction to Measure the Dynamic Properties of the Near Triad in Observers of a 3-D Display," Ophthalmic and Physiological Optics, vol. 22, pp. 385-388, 2002.

[4] A. Wilkins, "Visual Stress," Oxford Univ Press, 1995.

[5] J.-Y. Son and B. Javidi, "Three-Dimensional Imaging Methods Based on Multiview Images," IEEE/OSA Journal of Display Technology, vol. 1, pp. 125-139, 2005 .

[6] K. Ukai and A. Kibe, "Counterroll Torsional Eye Movement in Users of Head-Mounted Displays," Displays, vol. 24, pp. 59-63, 2003.

[7] Y. Suzuki, Y. Onda, S. Katada, S. Ino, and T. Ifukube, "Effects of an Eyeglass-Free 3-D Display on the Human Visual System," Japanese Journal of Ophthalmology, vol. 48, pp. 1-6, 2004.

[8] S. Tsukuda and Y. Murai, "A Case Report of Manifest Esotropia after Viewing Anaglyph Stereoscopic Movie (in Japanese)," Japanese Orthoptic Journal, vol. 18, pp. 69-72, 1988.

[9] G.K. von Noorden, "Binocular Vision and Ocular Motility, 4th Edition", St Louis: C. V. Mosby company, 1990.

[10] ISO, "Image Safety - Reducing the Incidence of Undesireble Biomedical Effects Caused by Visual Image Sequences," ISO International Workshop Agreement IWA3, 2005.

[11] K. Ukai, "Visual Stress Caused by Viewing Stereoscopic Motion Images: Background, Theories, and Observations," Displays, in press, 2006. 